\title{
The Design Model for Robotic Waitress
}

\author{
Jiaji Yang ${ }^{1}\left(\mathbb{D} \cdot\right.$ Esyin Chew $^{1}$ (D)
}

Accepted: 2 January 2021 / Published online: 25 January 2021

(C) The Author(s) 2021

\begin{abstract}
With the rapid development of traditional industries, intelligent robots have been widely used in the hospitality industry. Although the development of intelligent robots faces a positive trend and a good market in the hospitality industry, it also faces the problem that robots cannot effectively collect and use user data in the field of human-computer interaction. It not only affects the interaction experience between users and robots, but also prevents companies from getting valuable feedback in a timely manner. In order for intelligent robots to effectively utilize interactive information, the user experience of robot entertainment is improved. This paper proposes and establishes a basic technical model called iRCXM. Combining the iRCXM model with a decision tree classification algorithm is excepted effectively improve the interaction experience between humans and robots in hospitality. This paper designs a model of intelligent robot based on decision tree algorithm. The model divides the user into three sections, each corresponding to a different standard function. Using a decision tree classification algorithm model is excepted effectively judge users' current stage and whether they can move to the next stage. When the user reaches the final stage, it proves that the user has obtained a good interactive experience. At the same time, for users at different stages, the model will provide strategies for downward transformation so that companies can adjust and improve existing problems in a timely manner. In addition, the research developed a robot user interaction system based on the existing technology. The system is based on Android. Using HTTP protocol and Baidu Cloud AI API to realize simple face recognition and Sanbot-OpenSDK to implement simple robot control, the development of this system is to verify the feasibility of the model. The developed samples were tested in a real environment and feedback from customer experience was collected through semi-structured interviews. Finally, the feasibility of the model is verified.
\end{abstract}

Keywords Decision tree classification $\cdot$ Sanbot $\cdot$ Ircxm $\cdot$ Robotics $\cdot$ Humanoid robots

\section{Introduction}

There is research that explores expectation of social robots and how robots can serve as a companion [7]. There are some service robots currently working in China and Malaysia for food ordering and delivering [11,14]. However, these service robots are not as intelligent as human waiters or waitresses for enhancing customers' experiences and retaining customers, for instance, returning customers' recognition with personalised eating patterns, nutrition and favourite rec-

Supplementary Information The online version contains supplementary material available at https://doi.org/10.1007/s12369021-00745-1.

Jiaji Yang

JYang@ cardiffmet.ac.uk

1 EUREKA Robotics Lab, Cardiff School of Technologies, Cardiff Metropolitan University, Western Avenue, Llandaff, Cardiff CF5 2YB, UK ommendation. This research will respond to the needs and to enhance the customer experience. Its purpose is to research and design a humanoid robotic waitress for customer service to improve the traditional industries such as customer recognition with personalised recommendation, customers entertaining and engaging; payment receiver and emotion analytics. This study will use semi-structured interviews to collect data, and summarize the current situation of using intelligent robots as service personnel, and identify challenges and risks. In order to further solve these challenges and improve user experience, this research will design and develop a prototype of robot based on customer maturity model. The research envisages the development of an intelligent multi-lingual humanoid robot framework based on the results obtained from the above methods. The original intention of introducing intelligent robots is to better attract customers and reduce the cost of employees. Although robot waiters do not need emotion or training, they can not com- 
municate freely with customers, and their shortcomings are obvious [16]. Robots should concentrate on the development of data analysis and service intelligence. Intelligent technology has brought many challenges and problems while changing traditional industries. How to predict and solve these problems more reasonably is a major challenge for intelligent robotics. This research will focus on the challenge of introducing intelligent technology and develop a framework for the application of intelligent robots in traditional industries.

In addition, Fang et al. [8], based on the two theories of Ortony,Clore,Collins (OCC) emotion model and psychologist Ekman's emotion classification, set up a robot emotion model.The model is designed through a series of generic parameters to achieve the purpose of applying models to various environments, such as imaginary game characters and intelligent toys. In addition, the emotional space design is also introduced, and a system is designed in emotional space. Inspired by its model, this research designed and established an intelligent Robot for Customer eXperience Maturity (iRCXM) model for hospitality enhancement. The feasibility of the robotic application in combination with the iRCXM model was verified in real scenarios.

\section{Related Works}

It is noteworthy that the study of robots in hospitality is very new and scarce, mainly descriptive, forward-looking, or speculative [9]. Therefore, new problems and challenges will be brought by the application of intelligent robots. Leung [10] pointed out that stakeholder-defined new hospitality owners revolve around revenue growth, service customization, operational efficiency, and indoor automation. Since there is a big gap between stakeholders and academia in the definition of the intelligent hotel, stakeholders do not know where to start when managing the intelligent hospitality industry. Caleb Solly [3] believed that users can also help robots when robots help users; meanwhile, users can give feedback to optimize the system. The feedback reflects not only the optimization of the robot system but also the satisfaction of customers. Chung's [6] study indicated that hotels in the hospitality industry want to collect customer feedback in real-time to immediately disseminate positive feedback and respond to unsatisfactory customers while they are still on the scene. Guests want to inform their experience without affecting their privacy. Stakeholders in the hospitality industry hope that intelligent robots can interact more with users. Besides, Rodriguez [15] concluded that the optimal distance between users and robots is $69.58 \mathrm{~cm}$. Specifically, interaction with a certain greeting mode can attract users to maintain a longer interaction time; robots with the active search are more attractive to participants. The interaction time is longer than that of passively searching robots, suggesting that robots should be designed to keep at a certain distance from humans and consider adding the ability to allow robots to actively identify customers and attract them. Bowen [1] claimed that robots will be an important asset for many hotel organizations by the 2030s. The demand for robots will be partly driven by labor shortages, as well as the need to communicate with an increasing number of international travelers and create memorable customer experiences through the effective use of data available to customers. The introduction of robotics technology will be a major interruption in the industry. Since success during this interruption will require redesigning service delivery systems, robots can create customer value with better products, lower prices, or both. Service delivery redesign will also maintain a highlevel Customer Service [1].

Besides, studies by Bowen [1] and Ivanov [9] have indicated that young people are more receptive to the novelty of intelligent robots than older people. From another perspective, this suggested that the promotion of intelligent robots is very significant. The intelligent robotic hospitality industry will probably be widely popularized. Nowadays, most of the robots used in the hospitality industry are human and humanoid. However, through the analysis of a sixdimensional customer willingness table, Lu [12] revealed that the closer the robot is to humans, the more disgusted and feared it will be by consumers. This demonstrated that we should not choose robots that are too similar to humans when choosing robots. Conversely, some cartoon or animallike robots can stimulate humans to feel comfortable about them. People are more willing to accept robots like pets than real people. In the case of Nakanishi's research [13], most participants admit that interacting with robots can produce positive responses. However, some participants claim that the hotel does not need such a system, which may be related to a value-added but not essential service. Humanoid robot interaction can increase customer satisfaction. For example, the warm attitude of humanoid robots can provide customers with a warm experience. Personal identification technology may be an essential key to a better service system. Women prefer to interact with robots. The verbal interaction of voice control speakers has a negative impact on service evaluation [13]. The research was limited and the researchers did not take advantage of the ability of robots to collect data for analysis and feedback, even though the results of the study were negative. The simple greeting model in the study did not give customers more knowledge and experience of robotics. This explains from another perspective that the service intelligence of the robot should be more biased towards data collection and analysis and decision making. In addition, when robots provide services to humans. The ethical issues of robots are always the first concern. Should robots driven by artificial intelligence be responsible for their actions? Dr. 
Rob van den Hoven van Genderen [18] once pointed out in research that even if the autonomous system passes the Turing test, it will not incur legal liability. The function of the robot can produce legal effect, of course it represents the legal will of the initial legal participants and even the derived legal will. We better use our electronics or servants based on better technology to assist us in actual tasks. The smarter the system, the more trustworthy its functions. This view provides some support for the reliability of the experiments in this study.

\section{Research Methodology}

\subsection{Research Philosophy and Design}

The research ontology is post-positivism and the research philosophy of this research is phenomenology. Phenomenological studies have shown that this phenomenon can only be truly understood and experienced through subjective immersive research involving researchers directly. In phenomenological research, the interaction of researchers is an integral part of the research. Qualitative research methods indicate that the study needs to summarize a large number of existing literature. For intelligent robots operating in the hospitality industry, a large amount of case studies and experience will guide the future development of intelligent robots. Treatments and models from a large number of cases are generally considered to be mature. Therefore, the research and design of the new framework needs to be supported by these qualitative data. According to Robert Yin's "Case Study Design and Methods" [20], case studies are considered a suitable research method because the research questions are intended to discuss the context and causes of the various situations in the case. In addition, the goal of the study was to develop a robotic prototype program. In summary, the research design is mainly divided into two aspects. First, compare and analyze the cases interviewed and studied using comparative case studies to find out the available conclusions. Secondly, using the design research method and the conclusions obtained, the robot prototype program can be developed by artificial prototyping, which can be applied to the humanoid robot based on the user maturity model.

The second section of this article has obtained some conclusions through case studies and literature abstracts. These conclusions indicate that intelligent robots play a role in improving the user experience in the hospitality industry. A reasonable Human-Robot Interaction (HRI) model can effectively improve the user experience. However, in the existing cases, there is no technology or means to talk about or use the user maturity model to enhance the customer experience. The researchers conducted semi-structured interviews with a cafe manager. The Restaurant is located in Cardiff, UK, and the cafe also is selected as our research pilot test location. The interview focused on whether the introduction of smart technology has effectively improved traditional business models and increased industrial profits. On the other hand, the concept of the customer maturity model is presented to the manager during the interview. Interviews can provide strong support for the application and improvement of mature models. Questions about customer maturity include whether managers really understand the customer maturity model, whether they use the customer's mature model to improve the user experience, and what methods smart restaurants use to improve the customer experience.

\subsection{Customer's Mature Model}

Sitecore is a customer experience management company that provides web content management and multi-channel marketing automation software. Mature experience and a reliable architecture for assessing customer experience maturity. Grounding on Sitecore [17] Customer's mature model (shown as Fig. 1), this study proposes a customer experience maturity model for intelligent robots in the hospitality industry, namely intelligent Robot for Customer eXperience Maturity (iRCXM) model for hospitality (shown as Fig. 2). The proposed model is combined with the actual case of intelligent robots in the hospitality industry.

iRCXM is mainly divided into three parts, seven stages. The corresponding stage is used to judge the stage of the customer, and the customer is gradually transformed from the Initiate state into the Lifetime customer according to the transition conditions of each stage to enhance the user experience.

\subsubsection{Attract}

This section is mainly used for the acquisition of new customers, obtaining customer information and increasing customer value in preparation for the next part of the experience maturity improvement. This part is divided into three phases.

Initiate: The main criterion for this stage is whether the customer knows the merchant's website, how to navigate to the website, whether the website is satisfied with the content provided, and whether the merchant has the customer's email. For merchants, the key performance indicators (KPI) for this phase are website traffic and content usage. The main way for merchants to acquire new customers is often from the promotion of the network, the updating of the content and the timely push. Therefore, the above features are the basis for merchants to acquire new customers.

Radiate: When the customer meets the conditions of the first phase, they can convert to that stage. At this point, the merchant will provide customers with more mobile social 
Fig. 1 Customer experience mature model [17]

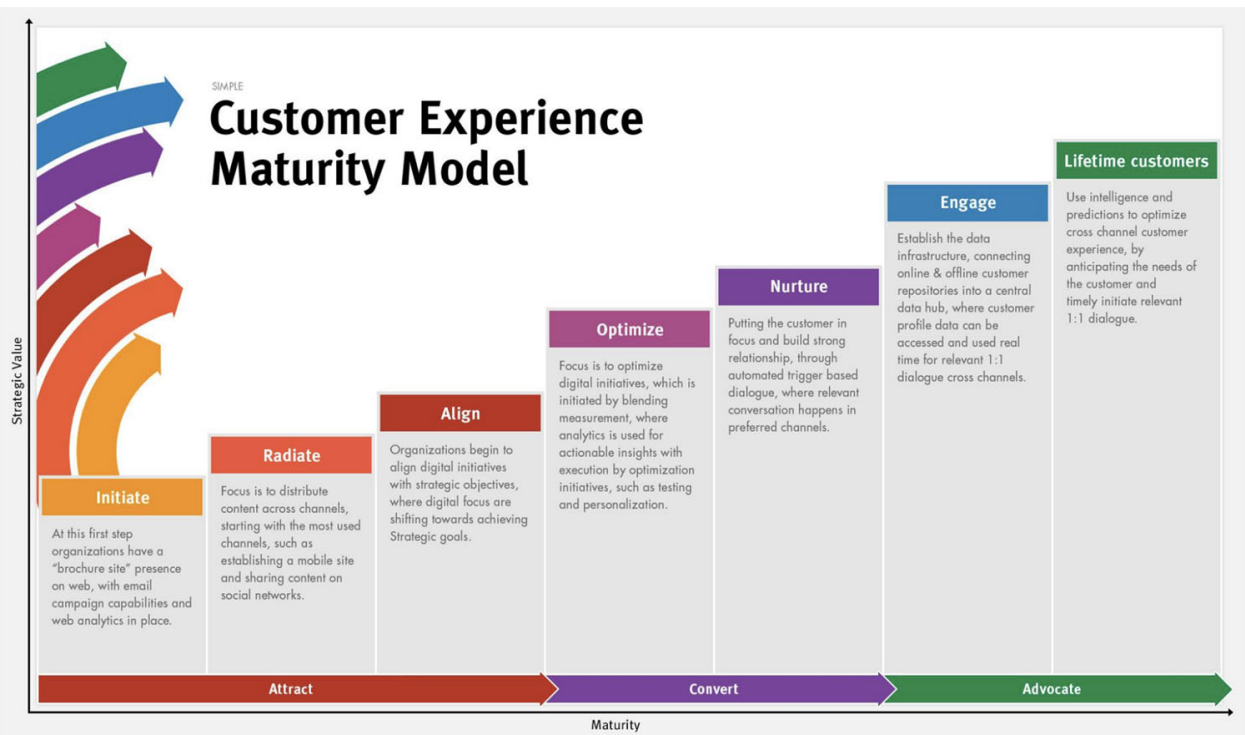

media channels in addition to email. Such as Facebook, Twitter, etc. The information and content of these channels can often be collected and analyzed. Customers can evaluate the products and services posted by merchants at these channels. These evaluations can generate greater value through emotional analysis. Therefore, the KPI at this stage is an analysis of websites and channels.

Align: When the user satisfies the characteristics of the above two stages, the quality of the customer is evaluated at this stage. The main evaluation criteria for customer quality is the customer's access value. The main calculation method of the visit value is: the number of times the customer browses the website + the number of comments / the number of times the customer visits the physical store + the number of consumption. The value of the visit mainly determines the effectiveness of various channels to obtain customers, and timely judges the satisfaction of customers with different channels, thereby improving the customer experience. The KPI at this stage is the value of customer visits.

\subsubsection{Convert}

This section focuses on deeper changes to customers with high access value. In order to better improve the user experience of this part, this part mainly focuses on providing personalized services for users.

Optimize: Improve your key customer experience with testing and personalization. Monitor and optimize content. And collect better customer intelligence data. At this stage, merchants should be memberized for key customers with high quality. And build a community of members to share information between members. The preferential policies offered by members should use at least $\mathrm{A} / \mathrm{B}$. At this stage, the member's personal information should be further col- lected, and the use of robots or related technology to track user behavior and establish a database of user behavior. The main KPIs at this stage are the satisfaction level of personalized marketing activities.

Nurture: This phase enhances the customer experience by linking more channels and increasing overall conversion rates. The main implementation of customer-centric triggerbased dialogue, collecting explicit and implicit customer behavior, automated processes. The main criterion for this stage is whether the customer has accepted the entry of the face recognition function provided by the intelligent robot. The introduction of face recognition enables businesses to quickly identify customer identities and customer preferences, and act accordingly based on their preferences and data to deepen their personalization.

\subsubsection{Advocate}

The main goal of this part is to turn users in the Nurture stage into Lifetime customers.

Engage: Connect different online and offline customer databases to support a single view of the customer. It is popular to say that this stage will provide customers with a recommendation system based on machine learning. Old users can be recommended for new products or activities based on their preferences. The criterion for this stage is whether the customer has a positive experience evaluation of the recommendation system. Negative evaluations of customers will be promptly feedback to the merchants and adjusted. A high-quality recommendation system that combines face recognition enables users to experience an unprecedented experience.

Lifetime customers: When users meet the criteria for all of the above stages, they can be considered as lifetime cus- 
Fig. 2 Intelligent Robot for Customer eXperience Maturityi $R C X M$ Mode

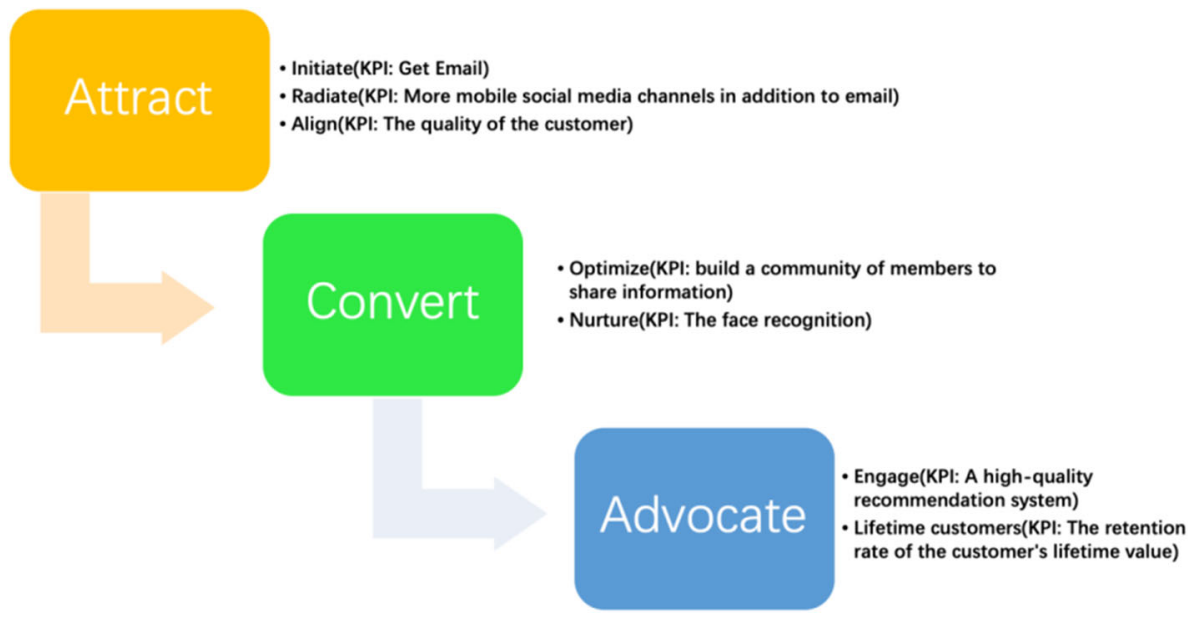

Fig. 3 Flow diagram for the design of sanbot service intelligence
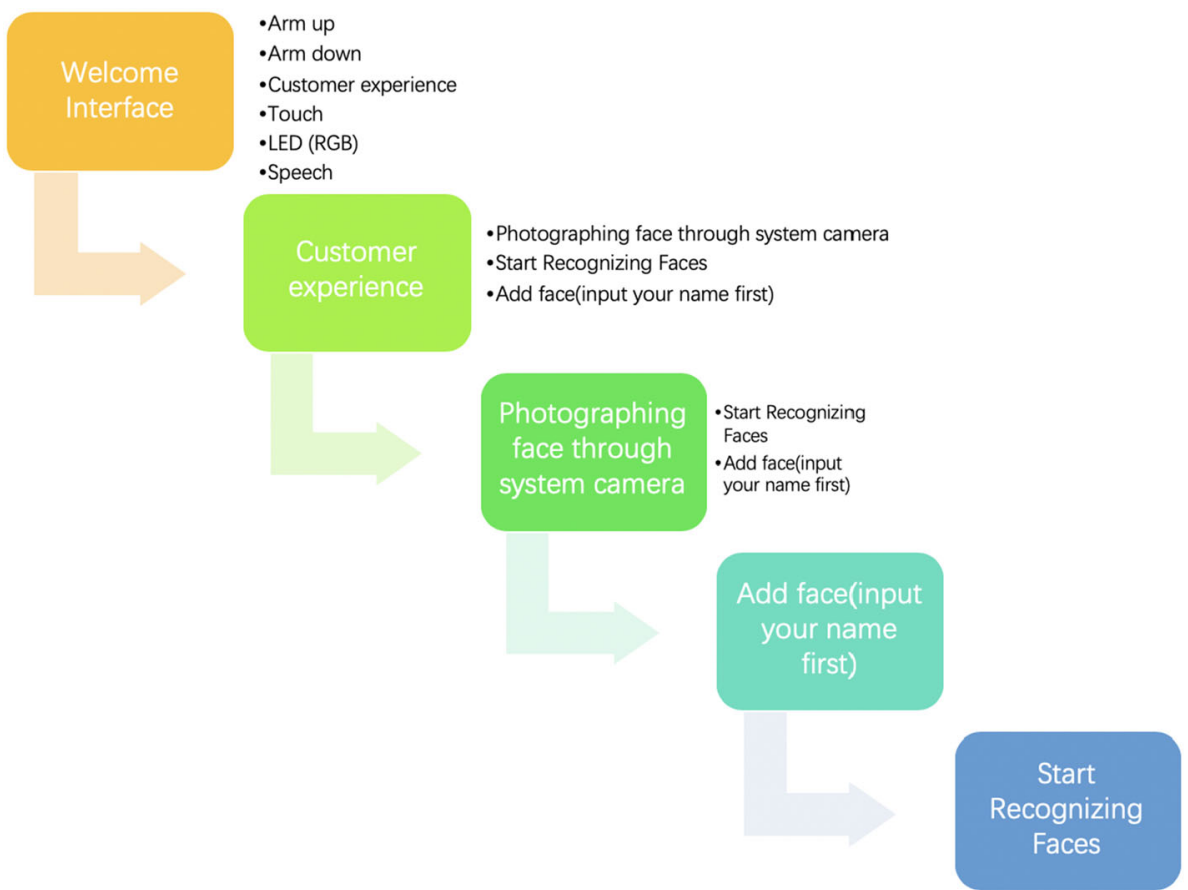

tomers. The KPI at this stage is the retention rate of the customer's lifetime value. The merchant will predict the behavior of the customer based on the data collected at all stages above. Customer preferences and behavioral data are constantly being updated to be continuously learned and used by the model.

\subsection{Service Intelligence Method}

According to the basic structure of the above framework. The decision conditions at different stages in the framework can be considered features. And ask the merchant to collect and build a user behavior database. The above framework clarifies that the ultimate goal of the user is to become the life customers of the merchant. Merchants want to be able to predict whether different customers can become Lifetime customers and choose how to improve the user experience based on predictions. This type of problem is more like a decision tree classification problem in machine learning. In addition to the face recognition and similarity-based recommendation systems mentioned in the framework. Classifying the user's decision tree allows the merchant to keep abreast of customer dynamics and make timely adjustments to the policies in the framework.

The algorithm of decision tree learning is usually a recursive selection of the optimal features, and the training data is segmented according to the features, so that each sub-data set has a best classification process. This process corresponds to the division of the feature space and also corresponds to the construction of the decision tree. The decision tree is used 
to divide the criteria, i.e. the features, at different stages of the iRCXM . Target whether customers are converted to life customers. The initial idea of the study was to calculate the entropy values of the different features. Entropy is defined as the expected value of the information. The probability of being able to become a lifetime customers is finally predicted based on the calculation of entropy.

\subsection{Service Intelligence Design with Sanbot}

This research designs corresponding service intelligence using Sanbot robot. The flow chart is shown in Figs. 2 and 3. Customers can command robots to raise or lower their arms, introduce themselves, turn on the lights and change the color of LED lights in the welcome interface. In addition, customers can enter the face recognition system through this interface. Customers take pictures of their own facial information by calling the camera of the robot. The captured images need to be added to the face database for recognition. In the process of adding to the face database, customers can set their own identity information such as name. Next time customers do not need to add faces repeatedly, they just need to take pictures and start recognizing. When the recognition is successful, the robot will say your name and welcome you. This model of robot is used later in the experiment of the service robot intelligence framework.

The Sanbot robot used in this study is a platform robot with customizable scenes for home (consumption, entertainment, education) and commercial (marketing, service, content). It is the pioneer of humanoid robot designed by Qihan Technology Co., Ltd. Sanbot Robot integrates the advanced research results of artificial intelligence all over the world, combines Internet cloud computing and Internet of Things (IoT) technology, and continuously improves the human-computer interaction experience and the home and commercial value of the robot through in-depth learning, so as to meet the platform-based robots that provide different intelligent services in different family and business scenarios.

\section{Findings, Pilot Implementation and Results}

\subsection{Framework for Intelligent Robotics for Hospitality}

Consolidating the case study and policy analysis results of Sect. 2 and the results of the smart restaurant survey. This study presents the Framework for Intelligent Robotics for Hospitality (Fig. 4).

Traditional entertainment industry wants to develop in the direction of intelligence. First, it needs to divide its own business processes. The business process is divided into entertainment, service, delivery, payment, service feedback and resources. The traditional hospitality industry needs to consider which businesses to add intelligent robots to. For example, the traditional hospitality industry mentioned in the case faces challenges in hosting foreign guests, so multilingual humanoid robots should be applied in hospitality. In addition, the use of the delivery robot needs to be determined in conjunction with the actual situation of the merchant. The results of the interviews and case studies show that the robot often needs to consider whether the item is suitable for robotic transmission when receiving and delivering items. Cases and interviews clearly indicate that the items being transferred need to be classified in detail. In addition, a large number of cases show that the addition of new technologies will inevitably lead to the renewal of payment systems, so it is indispensable to consider the intelligentization of payment systems. The case shows that the merchant hopes to get feedback from consumers in time with the help of the robot, and hopes to be able to process the feedback while the consumer is on the spot. So the robot should have a customer feedback system.

After dividing the traditional business process, the assigned business should be assigned responsibility. According to the policies of different countries and regions. The violation of the law by the robot shall be the responsibility of its responsible person. The robot after the task division should be assigned the corresponding manager. The way managers and robots can be distributed can be in the form of small groups, for example, three delivery robots are assigned a person in charge. The person in charge should assume the obligation of robot task completion, error task correction, robot maintenance and management.

Businesses should consider data protection measures when collecting data with service intelligence and robots. Most cases show that consumers don't want their data to be leaked. This study considers the encryption of key data of users, the leakage of user-critical data, and the data acquisition of user behavior needs to inform the customer and ask for their consent. In addition, network information security and data protection laws and related policies need to be considered in the process of collecting data using robots. Ensure that the rights of merchants and consumers are not violated.

On the basis of sound security measures, interactive models should be designed to enhance the consumer experience. This framework combines iRCXM to improve the user experience and capture user data (Show as Fig. 2).

Use the HRI interactive mode specified by iRCXM to select the appropriate service intelligence technology. This study believes that merchants should use decision tree classification as the core algorithm, recommendation system, and face recognition to help develop a service intelligence system that can respond to consumers in a timely manner and enable merchants to get timely feedback. The results of the decision tree classification model can be used to improve the 
Fig. 4 Intelligent robotics for hospitality framework

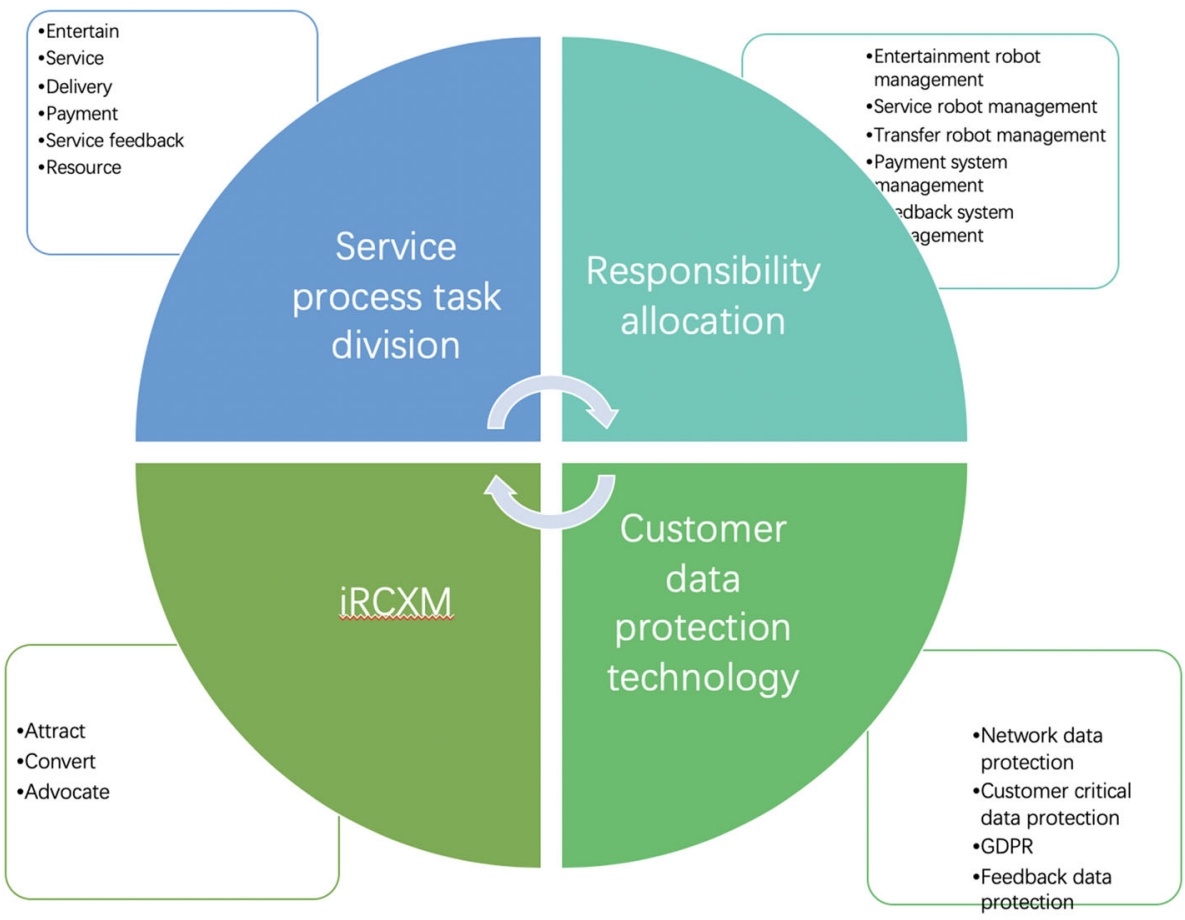

user experience and enable the merchant to develop marketing strategies in a timely manner.

\subsection{Robot EURKEA Gen-1 Service Experiment}

Grounded on the proposed framework (Fig. 4), the first intelligent Service Robotic Waitress in Wales, namely Robot EURKEA Gen-1 is designed and piloted. Robot EURKEA Gen-1 has been worked in a coffee shop called Mrs Jones Cater in Cardiff, Wales, and interviewed customers who had experienced robots using semi-structured interviews (Fig. 5). Please see the following tweets and videos for the pilot implementation:

(1) 316 Home café (2019) [2]

(2) Mum \& baby, children, Cardiff Uni \& Bristol Uni students, adult customers had fun with Robot EUREKA GEN-1's exciting features developed by a brilliant master student, [4]

(3) Prep for the pilot test of the Robot EUREKA GEN-1, the first Robotic Waitress in Wales at Mrs Jones Cater.[5]

This study designed two main user interaction interfaces for the robot. In the welcome interface, the user can simply control the Sanbot through the buttons on the touch screen (Fig. 6). These controls include raising the arm, lowering the arm, changing the color of the LED light on the body, and synthesizing the voice for self-introduction. When you click the Customer experience button, the page will jump to the face recognition interface (Fig. 7).

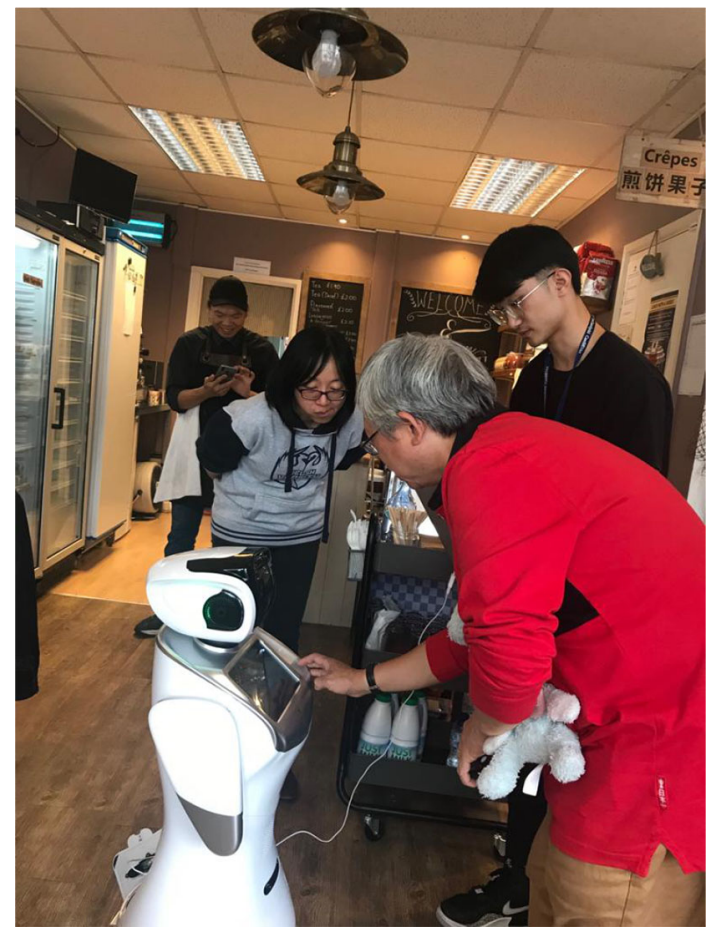

Fig. 5 Robot EUREKA Gen-1

In the face recognition interface, new customers can choose to add faces to the face database and enter names. Old customers can choose to perform face recognition directly. After the recognition is successful, the customer's name will 


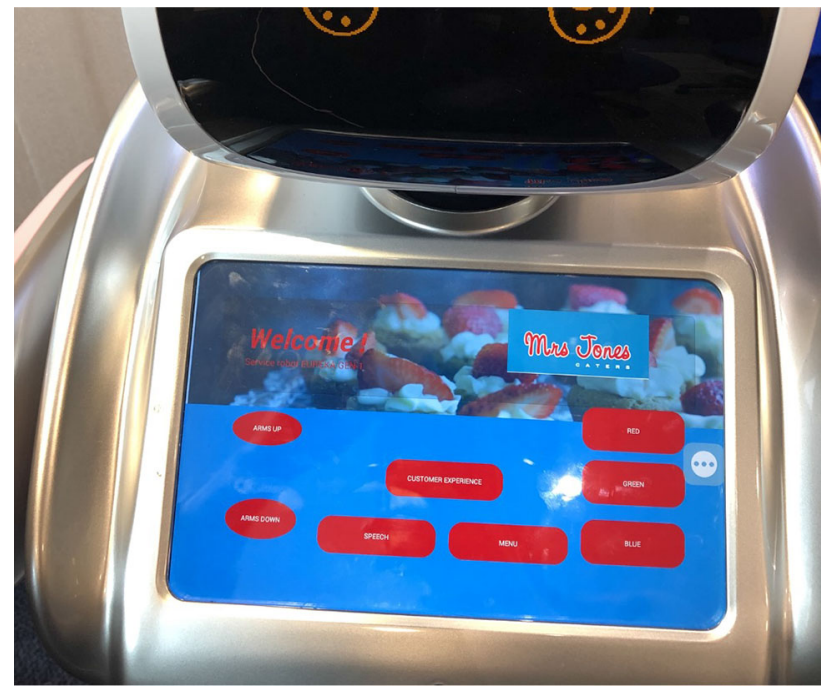

Fig. 6 Welcome interface

be displayed on the recognition interface, and the robot will say welcome back + name.

In addition, customers can experience applications supported by the Sanbot robot itself, such as simple question and answer, turn on the projector, sing, dance, play movies, follow customers, games and more. The sensor on the Sanbot robot can feel the touch of the customer. There are different greeting patterns when customers touch their arms, shoulders and head.

Semi-structured access is divided into two parts. Before the customer experiences the robot, it collects some basic information, such as age, occupation, the experience of being served by the robot, and so on. After the customer has finished the experience, the researcher will continue to collect feedback on the customer experience. The content includes whether you feel that the robot really attracts you, whether you can accept interaction with the robot, and whether it is safe to hold customer information for the robot (such as DSample of Primary Data collection).

In fact, the research design and development of the customer's ordering interface based on store information (Fig. 8), but due to some technical problems failed to successfully run on the robot. This is also the shortcoming of this study.

This experiment was designed in accordance with framework for intelligent robotics for Hospitality. The robot has an interface that interacts with the user, and the robot can collect user information and data. Face recognition uses cloud technology provided by Baidu. Use the Http request sent to the cloud server to upload and identify the customer's face information. The face database encrypts the access rights by using an encryption algorithm to ensure the security of the user data. In addition, throughout the process, the researcher

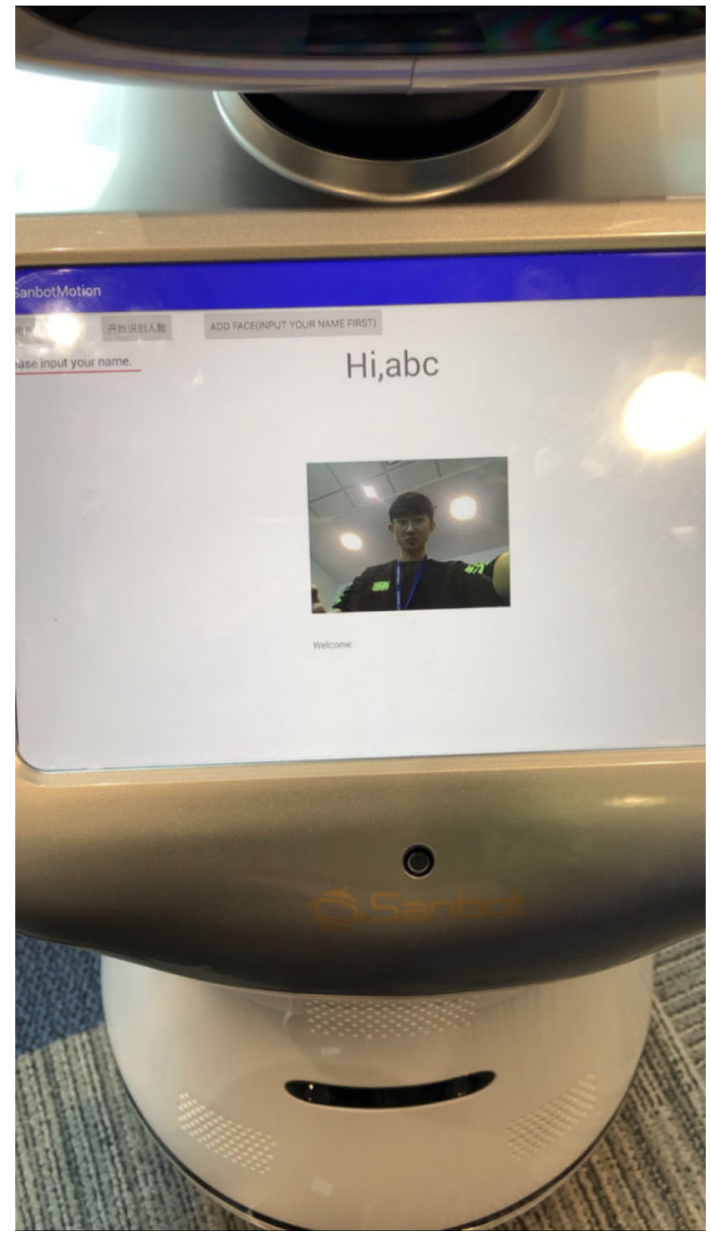

Fig. 7 Face recognition successful interface

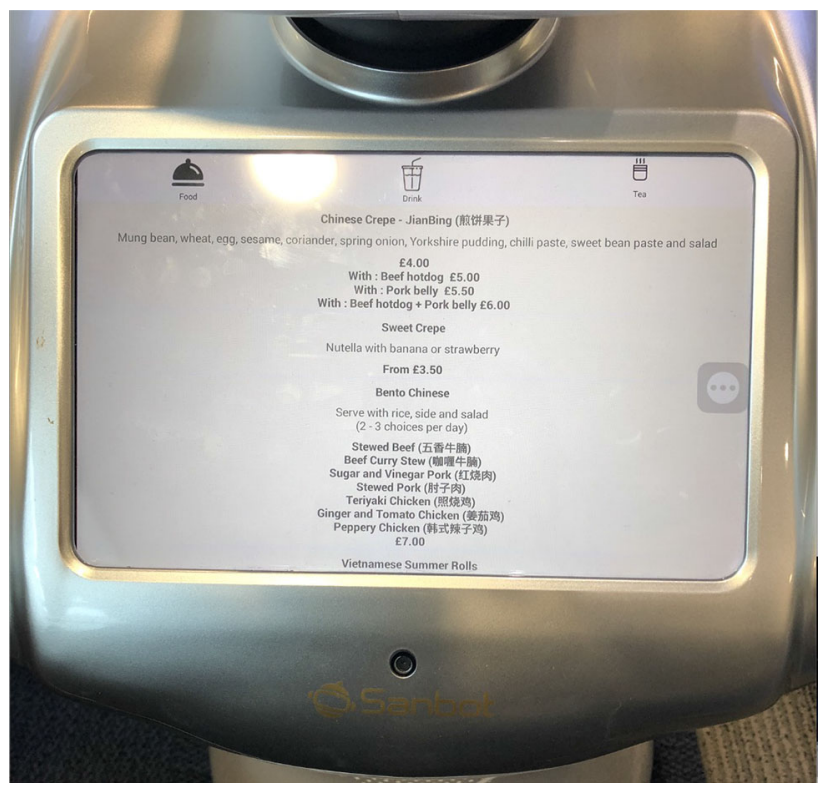

Fig. 8 Ordering interface 
is responsible for supervising and correcting the activities and behavior of the robot.

\subsection{Sanbot Technical Shortcomings}

During the test, the researchers found that the robot's sensor could not sense the baby's touch screen. This causes the baby to evade or stop the robot. The researchers believe that the baby is at risk of interacting with the robot, so a guardian is required to monitor and avoid accidents when interacting. In addition, some older people think that the robot is not high enough. This makes it very inconvenient for older people to be interactive. Therefore, the robot should be more userfriendly when designing. The robot's screen can't sense the touch of the nail, and some women may not be able to easily master the interaction skills at the beginning. In addition, when the robot performs face recognition, the researchers found out that the robot can accurately recognize that the face is affected by the lighting factor of the restaurant. The dim light when collecting faces can make the robot unable to recognize them correctly.

\subsection{Insufficient User Experience}

It is often unclear what the robot can do when interacting with the robot, which causes the customer to be hindered while interactive. Researchers believe that adding operational hints to the robot interface is more conducive to user interaction with the robot. In addition, 8 of the 12 customers who experienced the experience proposed whether or not the ordering operation can be performed on the robot. This proves that the robot needs to have the corresponding functions in the current environment in different hospitality environments. This also reflects from another perspective that customers prefer to be hosted by robots rather than service personnel.

The final feedback on the user experience is generally positive. Twelve customers have read about robots in news or in newspapers or on TV. But 12 customers have indicated that they have never been served by robots. This shows that robots have great potential in the hospitality industry. In addition, 8 out of 12 customers believe that robots really help the hospitality industry. These eight customers have emerged their expectations for the future development of service robots. Three of the other four customers said they were not sure if the robot could really help the hospitality industry. The main reason was their lack of understanding of the technology and development status of the field. One customer showed a strong rejection, and $\mathrm{He} / \mathrm{She}$ showed that he was worried that future robots would replace their work. When talking about whether the robot attracts them, there are 9 customers who indicate that they are attracted by the robot. The main reason for being attracted is the curiosity of the robot. To experience the new technology, the robot is very cute, and the robot can save money. One of the customers said that they did not like robots that were too similar to humans. Excessively similar robots would cause customers' own dislike and fear. This is also consistent with the view put forward by $\mathrm{Lu} \mathrm{[12]} \mathrm{in} \mathrm{the}$ case study of the second chapter of this study.

\section{Conclusion and Recommendation}

This study demonstrates the feasibility of the framework for intelligent robotics for hospitality described in this study by using a large number of case studies and experiments. This study summarizes the current problems of intelligent robots in the hospitality industry mainly in terms of HRI, user experience, data confidentiality and effective service intelligence. The existing problems are analyzed and proposed. The iRCXM model is proposed to improve the user experience. The feasibility and development potential of the model combined with decision tree algorithm are proved by interviewing customers. In addition, the study also found that robots that are too similar to humans can cause panic among customers. The design of the robot should be more human. Experiments show that robots with service intelligence have positive development potential in the hospitality industry. Different hospitality industries only need to follow the framework of the research to complete their conversion to smart entertainment.

Future work will involve more research and design on iRCXM models, applying AI technical thinking (such as decision tree classification or recommendation systems), visual and image sensing, and tactile sensors to automatically capture customer satisfaction, in-store user experience and emotions analysis. More natural language conversation between service robot and customers are recommended. As the development of the restaurant industry is global and diversity, consumers may come from different countries and have different cultural backgrounds. This means that the language used by consumers is often not single language but multi-language. For the traditional hospitality industry, this creates communication challenges. The future maturity of multi-language robotics provides a reasonable solution to this problem. Ethical consideration such as Tokku RT Special Zones [13] in Wales, methods of obtaining customers' consent with data privacy protection for robot-collected data are key agenda in the real world implementation.

Recommendation for the future work and next phase of empirical experiment

(1) More research and design on iRCXM models, applying AI technical thinking (such as decision tree classification or recommender systems with supervised learning). 
(2) Visual and image sensing, and tactile sensors to automatically capture customer satisfaction with emotion analytics.

(3) More natural language conversation between service robot and customers without time delaying. 5G communication and connection are recommended.

(4) Research on Multilingual Robot.

(5) Ethical consideration to apply Tokku RT Special Zones [19] in Wales.

Acknowledgements Funding was provided by EUREKA Robotics Lab of Cardiff school of technologies (EUREKA Service Robot Gen-1).

\section{Compliance with ethical standards}

Conflict of interest N/A-Consent obtained from all parties.

Open Access This article is licensed under a Creative Commons Attribution 4.0 International License, which permits use, sharing, adaptation, distribution and reproduction in any medium or format, as long as you give appropriate credit to the original author(s) and the source, provide a link to the Creative Commons licence, and indicate if changes were made. The images or other third party material in this article are included in the article's Creative Commons licence, unless indicated otherwise in a credit line to the material. If material is not included in the article's Creative Commons licence and your intended use is not permitted by statutory regulation or exceeds the permitted use, you will need to obtain permission directly from the copyright holder. To view a copy of this licence, visit http://creativecomm ons.org/licenses/by/4.0/.

\section{Appendix}

Part of the project source code: https://github.com/EUREKACardiffMet/pilot-test.git.

\section{References}

1. Bowen J, Morosan C (2018) Beware hospitality industry: the robots are coming. Worldw Hosp Tour Themes 10(6):726-733

2. Cafe (2019) Lunch with a robot. https://twitter.com/ SmartHomeCafe1/status/1194546188390940672?s=20. Accessed 6 Dec 2020

3. Caleb-Solly P, Dogramadzi S, Huijnen CA, Hvd Heuvel (2018) Exploiting ability for human adaptation to facilitate improved human-robot interaction and acceptance. Inf Soc 34(3):153-165

4. Chew E (2019) First service robot in wales. https://twitter.com/ EsyinChew/status/1176979159622574082?s=20. Accessed $1 \mathrm{Feb}$ 2020

5. Chew E (2019) Pilot test. https://twitter.com/EsyinChew/status/ 1176975535995572224?s=20. Accessed 1 Feb 2020

6. Chung MJY, Cakmak M (2018) "How was your stay?": Exploring the use of robots for gathering customer feedback in the hospitality industry. In: 2018 27th ieee international symposium on robot and human interactive communication (RO-MAN). IEEE, pp 947-954
7. de Graaf MMA, Allouch SB (2017) The influence of prior expectations of a robot's lifelikeness on users' intentions to treat a zoomorphic robot as a companion. Int J Soc Robot 9(1):17-32

8. Fang Y, Zengqiang C (2006) Establishing an emotional model based on artificial intelligence. Inf Control 36(6):673-678

9. Ivanov S, Webster C, Garenko A (2018) Young Russian adults' attitudes towards the potential use of robots in hotels. Technol Soc 55:24-32

10. Leung R (2019) Smart hospitality: Taiwan hotel stakeholder perspectives. Tour Rev 74(1):50-62

11. Loh I (2018) Meals on wheels at this kopitiam. The star newspaper. https://www.thestar.com.my/news/nation/2018/01/06/meals-onwheels-at-this-kopitiam-ipoh-outlet-hires-mechanical-staff-toimprove-service-efficiency/. Accessed 1 Feb 2020

12. Lu L, Cai R, Gursoy D (2019) Developing and validating a service robot integration willingness scale. Int J Hosp Manag 80:36-51

13. Nakanishi J, Kuramoto I, Baba J, Kohei O, Yoshikawa Y, Ishiguro $\mathrm{H}$ (2018) Can a humanoid robot engage in heartwarming interaction service at a hotel? In: Proceedings of the 6th international conference on human-agent interaction. ACM, pp 45-53

14. Robot H (2019) Haidilao: robots staff China's top hotpot chain. BBC news. https://www.bbc.co.uk/news/av/business-46946431/ haidilao-robots-staff-china-s-top-hotpot-chain. Accessed $1 \mathrm{Feb}$ 2020

15. Rodriguez-Lizundia E, Marcos S, Zalama E (2015) A bellboy robot: study of the effects of robot behaviour on user engagement and comfort. Int J Human-Comput Stud 82:83-95

16. Sanbo Z, Jia H (2019) "Nobody dining" has no way to go? The robot meets the waterloo! http://www.linkshop.com.cn/web/ archives/2019/417455.shtml. Accessed 26 Sept 2019

17. Sitecore (2017) Customer experience maturity model. http:// mediacontent.sitecore.net/webinars/CX_Maturity_Model_NA/ CX_Maturity_Model.pdf. Accessed 26 Sept 2019

18. van Genderen RH (2015) Machine medical ethics and robot law: legal necessity or science fiction? In: van Rysewyk S, Pontier M (eds) Machine medical ethics. Springer, Berlin, pp 167-177

19. Weng YH, Sugahara Y, Hashimoto K, Takanishi A (2015) Intersection of "tokku" special zone, robots, and the law: a case study on legal impacts to humanoid robots. Int J Soc Robot 7(5):841-857

20. Yin RK (2009) Case study methods: design and methods

Publisher's Note Springer Nature remains neutral with regard to jurisdictional claims in published maps and institutional affiliations.

Jiaji Yang is the Associate Tutor at Cardiff School of Technologies (CST) and the STEM Outreach Lead at EUREKA Robotics lab and a Ph.D. researcher at Cardiff Metropolitan University. He is currently dedicated to researching the application and development of humanoid robots in the hospitality industry.

Esyin Chew is a Reader in Robotics and Educational Technologies at Cardiff Metropolitan University now and an interdisciplinary academic working with humanoid robots that enhanced assessment and feedback in education and healthcare. Before joining Cardiff Metropolitan University, she was the Co-ordinator for the Master in Business Information Systems programme and a Senior Lecturer in IT at Monash University Malaysia. Esyin was an independent academic consultant prior to Monash, a Senior Lecturer in the Centre for Excellence in Learning and Teaching (CELT), University of South Wales and a Lecturer in the Faculty of Computer Science and IT, University of Malaya. Esyin is the founder of the EUREKA Robotics Lab [www.cardiffmet. 
ac.uk/eureka] at Cardiff School of Technologies, researching and designing humanoid robotics in education, hospitality, and healthcare. Demonstrating a track record of research and design leadership for cutting edge humanoid robotic programs, with AI capabilities. The UK Parliament Artificial Intelligence Select Committee published her expert viewpoint on the implications of AI, titled: "What are the implications of artificial intelligence? In Love and War" [https://bit.ly/3iC1QDC], as well as the UK Parliament Education Committee's Fourth industrial revolution [https://bit.ly/3lsyFoc] inquiry. 